\title{
Cost-effectiveness of predictive genetic tests for familial breast and ovarian cancer
}

\author{
NIKKI BREHENY, ELIZABETH GEELHOED, JACK GOLDBLATT \\ \& PETER O’LEARY
}

\begin{abstract}
Aim: To examine the relative cost-effectiveness of predictive genetic tests for familial breast and ovarian cancer provided by Genetic Services of Western Australia.
\end{abstract}

Methods: The relative cost-effectiveness was assessed using a decision analytic model.

Results: The cost and outcomes of genetic testing was compared in first-degree relatives of known BRCA1/2 mutation-carriers who have a $50 \%$ risk of carrying the mutated gene (intervention group) to individuals with the same a priori risk but who do not undergo a genetic test (control subjects).

Since genetic testing enables the restriction of intensive surveillance to individuals with an identified BRCA1/2 gene mutation, net savings in the period observed (age $25-70$ ) were $\$ 980-\$ 1008$ per woman in the ovarian intervention group and $\$ 1681$ $\$ 1795$ per woman in the breast intervention group, and delayed the onset of breast cancer (6mths BRCA1, 3mths BRCA2).

Compared to control subjects undergoing population surveillance, it was estimated the onset of breast cancer could be delayed at a total net cost of $\$ 3055$ (5.1yrs) to \$3389 (3.2yrs) for women in the breast intervention group with BRCA1/2 mutations. Since population surveillance is not currently recommended for ovarian cancer, control subjects undergoing no surveillance were compared with the intervention group. The onset of ovarian cancer was delayed at a net cost of $\$ 1630$ (3.5yrs) to $\$ 2509$ (1.2years) for women with BRCA1/2 mutations.

Conclusions: Testing allows targeted high-level surveillance for gene mutation carriers, which ensures the cost-effective use of resources and reduces cancer-related morbidity if clinical recommendations for intervention are adopted. 


\section{Introduction}

Inherited predisposition to cancer is thought to account for $5-10 \%$ of all cancer incidence ${ }^{1}$. Advances in genetic testing technology have many promising applications in health including improved diagnosis of disease and the earlier detection of genetic predisposition to adult-onset conditions, such as familial cancer. This will have important implications for resource allocation given the capacity to compare costs with associated benefits. Economic evaluation helps determine the relative value of new technology and enables better planning for the provision of future cancer genetic services.

In order to understand the relative cost-effectiveness of genetic testing the prevalence and penetrance of the gene mutation must be considered as well as the uptake and efficacy of available interventions to prevent or detect cancer early ${ }^{2}$. Reported benefits resulting from increased surveillance in women with a mutated BRCA gene have included earlier detection of breast cancer and an expected mortality reduction in women less than 50 years of age $\mathrm{e}^{3,4}$. There is also evidence that prophylactic intervention, such as bilateral mastectomy, has been associated with a reduction in the incidence of breast cancer of at least $90 \%{ }^{5}$. Though there is evidence to suggest oophrectomy reduces risk of breast cancer, it is not within the scope of this study.

The absence of reliable surveillance methods for the early detection of ovarian cancer, and the poor prognosis following symptomatic presentation, have prompted many oncologists to recommend bilateral prophylactic salpingo-oophorectomy after childbearing $^{6,7}$. Furthermore, studies have validated the prophylactic role of surgical intervention and provided a convincing rationale for genetic testing in women with a strong family history ${ }^{8,9}$.

This study aimed to evaluate the relative costs and outcomes of genetic testing for familial breast and ovarian cancer through Genetic Services of Western Australia (GSWA). The investigation included familial breast and ovarian cancers suitable for predictive DNA based testing on the basis of inherited BRCA 1/2 mutations. The theoretical cohorts simulated asymptomatic first-degree relatives of individuals with a known BRCA1/2 mutation, who had a 50\% chance of inheriting the cancerpredisposing gene mutation.

Since reliable age and gene-specific cancer mortality data were not available at the time of modelling for relevant population subgroups, the impact of genetic testing and increased surveillance on mortality was not explored in this study. Instead the study focus was confined to the impact of genetic testing and increased surveillance on reduced cancer morbidity and, accordingly 'cancer-free years' was the most appropriate method to measure and report reduced cancer morbidity.

\section{Methods}

\section{Models}

Economic decision modelling software (TreeAge Data ${ }^{\mathrm{TM}}$ version 4.0) was used to develop a decision-analysis model, which mimicked the course of testing and treatment for women entering the Familial Cancer Program at GSWA. The attendance of women at high-risk of inherited breast or ovarian cancer in the program was 
adequate to warrant this study. Previous studies from which much of the data is derived also indicated women in this risk group merited further study.

Internet based searches were conducted using PubMed, Medline and Ovid on the costeffectiveness of genetic testing, cancer surveillance and surgical intervention to reduce cancer morbidity.

Markov models were used to predict the age of breast and ovarian cancer onset and costs of surveillance for carriers of a mutated BRCA1/2 cancer gene compared with non-mutation carriers and control subjects.

Each Markov cycle represented one year. The age and penetrance (or likelihood a person will develop cancer) relating to each cycle governed what proportion of women stayed in the 'well' state or shifted into the 'cancer' state. See Figure 1.

\section{Figure 1: Predictive genetic testing decision model for persons at high risk of} familial breast cancer

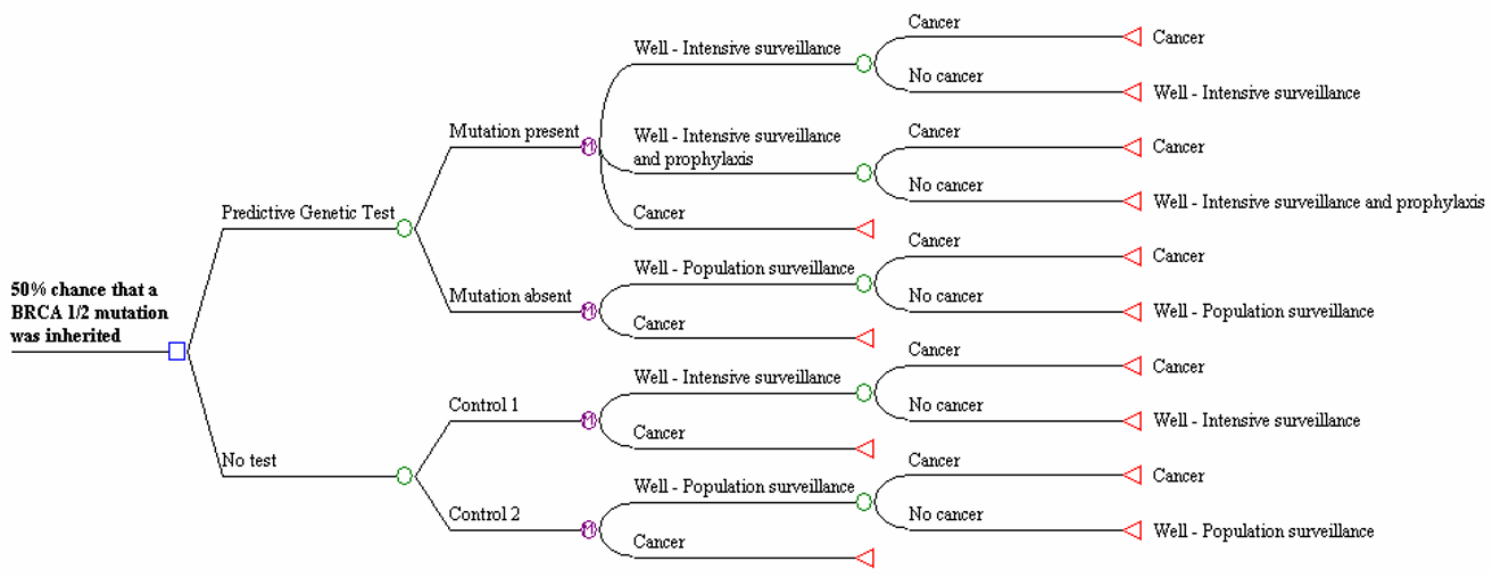

Surveillance strategies modelled in this study (See Table 2) were based on the National Health and Medical Research Council (NHMRC) clinical practice guidelines for the detection and treatment of familial cancer ${ }^{10,11}$ and corroborated by Western Australian (WA) surgeons and oncologists working in the area. Accordingly, the models cover the period from age 25 to 70 .

All women in the intervention group had predictive genetic testing to determine their mutation status. Inherited cancer segregates as an autosomal dominant trait, thus the offspring of a BRCA1/2 gene mutation carrier have a 1 in 2 chance of inheriting their cancer-predisposing mutation. Hence, half the women in the intervention group were deemed to be mutation positive and underwent intensive surveillance and prophylaxis as recommended. The other half were identified as mutation negative and only had population surveillance. 


\section{Control Groups}

Despite their high-risk family history, since control subjects did not undergo predictive genetic testing, their mutation status was unknown.

Women may or may not have increased their surveillance based on family history alone (perceived breast or ovarian cancer risk) so dichotomous scenarios were reviewed corresponding to surveillance extremes. For example, the intervention group (known mutation status) was compared with control subjects (unknown mutation status) having either intensive surveillance and prophylaxis, or population surveillance.

As a baseline for comparison we examined two scenarios corresponding to each extreme. The intervention group was first compared to control subjects (unknown mutation status) who adhered to clinical recommendations for increased surveillance based on their family history alone (control group 1). The intervention group was then compared with control subjects who have population or no surveillance, despite their high-risk family history (control group 2). The actual surveillance behaviour of control subjects was expected to be a mid-point between population surveillance or intensive surveillance and prophylaxis.

\section{Costs}

All costs are provided in Australian dollars and standardised to 2001-2002 prices using health index deflators ${ }^{12}$. Future costs were discounted at a rate of $5 \%$ per annum. Counselling, genetic testing, surveillance, surgery and treatment costs were based on patterns of care in WA and are consistent with NHMRC clinical practice guidelines for familial cancer ${ }^{13}$. Lifetime cancer treatment costs were taken from the Australian Institute of Health and Welfare ${ }^{14}$. Mean ages for surgery and cancer diagnosis were used to estimate years of discounting ${ }^{15,16,17,18}$.

Counselling costs were estimated in accord with GSWA and based on the average session time of Familial Cancer Program patients. Hourly costs were assigned based on staff and office requirements. Since diagnostic tests are required to confirm an index case before cascade testing is possible, a diagnostic cost component was also included.

Genetic testing costs were provided by the Molecular Genetic Laboratory, Princess Margaret Hospital. Cancer prevention and prophylaxis costs were provided by costing centres in Perth's major teaching hospitals based on a breakdown (pathology, medical, nursing, allied health and other) of surgical procedures in 2001-2002 and averaged to provide an estimate. Though capital expenditure was not assessed, a review of total WA health expenditure in 1999-2000 indicates this component would be less than $5 \%{ }^{19}$.

Indirect costs were not included. 


\section{Outcome data}

Outcomes on the effectiveness of cancer screening and interventions on cancer incidence in these high risk individuals were derived from the published literature. Age-related 'population risk' of breast cancer represented national rates in $2000^{20}$. To enable comparisons between the simulated cohorts breast and ovarian cancer were examined separately and the risks of developing the two cancers were assumed to be independent.

Cumulative age-related cancer incidence in mutation carriers, with and without the recommended clinical intervention, were gathered from previous studies and factored for attrition, and hence took account of all-cause mortality. The age-specific 'inherited risk' of breast cancer in BRCA1/2 mutation-carriers was based on $65 \%$ or $45 \%$ penetrance respectively by age 70 consistent with studies by Antoniou et $\mathrm{al}^{21}$. The age-specific 'inherited risk' of ovarian cancer in mutation-carriers was based on 39\% or $11 \%$ penetrance for BRCA1/2 respectively by age 70 , as previously reported ${ }^{22}$.

The simulated population represented offspring of known mutation-carriers (at $50 \%$ risk of inheriting the gene mutation) and assumed full compliance with NHMRC clinical recommendations for intervention ${ }^{23}$.

Interventions modelled on behalf of population or intensive surveillance and prophylaxis are listed in Table 1. For each intervention the optimal age, required frequency of an event and the non-discounted cost is reported.

Based on trends in WA, it was assumed that around $30 \%{ }^{24}$ of the breast intervention group would elect prophylactic bilateral mastectomy at a mean age of 38 years, with a lifetime reduction in breast cancer risk of $90 \%{ }^{25,26}$ since many women elect surveillance but no surgery, this option was examined within the model. Mammographic screening of women between 50-69 years has been shown to reduce their lifetime risk of breast cancer by $35 \%{ }^{27}$. Previous studies indicated that the younger age of cancer diagnosis in women with BRCA mutations justified screening from an earlier age $28,29,30,31,32$. On this basis it was presumed that mutation carriers aged 35 to 49 would also reduce their lifetime risk of breast cancer by $35 \%$ though mammographic screening and clinical breast examination.

The available screening measures for ovarian cancer, such as transvaginal ultrasound and serum CA-125, have limited sensitivity and specificity and may not reduce ovarian cancer mortality ${ }^{33}$. For this reason it was assumed that all women in the intervention group would undergo the recommended prophylactic salpingooophorectomy at age 40 and achieve a lifetime reduction in ovarian cancer risk of $96 \%$ as previously reported ${ }^{34,35}$. Sensitivity analysis was used to test the effects of varying these outcomes.

Complications from medical intervention, intangible costs and benefits were not incorporated. 


\section{Results}

Costs associated with genetic testing, surveillance, prophylaxis and cancer treatment are listed in Table 1. Estimated total surveillance costs are listed in Table 2.

Table 1: Intervention costs at 2001-2002 prices

\begin{tabular}{|c|c|c|c|}
\hline Intervention & $\begin{array}{r}\text { Age of intervention } \\
(\mathrm{yrs})\end{array}$ & Frequency of event & $\begin{array}{r}\text { Cost per event } \\
\text { (undiscounted) }\end{array}$ \\
\hline Genetic counselling + test, carrier & & once only & $\$ 1,012$ \\
\hline \multicolumn{4}{|c|}{ Ovarian Cancer Intensive Surveillance \& Prophylaxis } \\
\hline Transvaginal ultrasound & $35 *-40$ & $12 \mathrm{mths}$ & $\$ 129$ \\
\hline CA125 & $35-40$ & $12 \mathrm{mths}$ & $\$ 24$ \\
\hline Prophylactic salpingo-oophorectomy & 40 & once only & $\$ 7,216$ \\
\hline \multicolumn{4}{|c|}{ Breast Cancer Intensive Surveillance \& Prophylaxis } \\
\hline Clinical breast examination & $25-37$ & $4 \mathrm{mths}$ & $\$ 65$ \\
\hline Mammogram & $35 *-37$ & $12 \mathrm{mths}$ & $\$ 163$ \\
\hline Prophylactic bilateral mastectomy & 38 & once only & $\$ 11,547$ \\
\hline CT chest scan post surgery & 39 & once only & $\$ 175$ \\
\hline Clinical ex. of chest wall and lymph nodes & $39-70$ & $6 \mathrm{mths}$ & $\$ 65$ \\
\hline \multicolumn{4}{|l|}{ Breast Cancer Intensive Surveillance only } \\
\hline Clinical breast examination & $25-70$ & $4 \mathrm{mths}$ & $\$ 65$ \\
\hline Mammogram & $35 *-70$ & $12 \mathrm{mths}$ & $\$ 163$ \\
\hline \multicolumn{4}{|l|}{ Population Surveillance (breast cancer only) } \\
\hline Mammogram & $50-69$ & $24 \mathrm{mths}$ & $\$ 163$ \\
\hline Ovarian cancer treatment (life) & & once only & $\$ 19,735$ \\
\hline Breast cancer treatment (life) & & once only & $\$ 11,616$ \\
\hline
\end{tabular}

*Or 5yrs before youngest affected family member

Table 2: Total surveillance costs

\begin{tabular}{|lrr|}
\hline Intervention & No Discount & 5\% Discount \\
\hline Breast Cancer & & \\
Intensive surveillance and prophylaxis & $\$ 18,906$ & $\$ 9,785$ \\
Intensive surveillance only & $\$ 14,838$ & $\$ 6,493$ \\
Population surveillance & $\$ 1,630$ & $\$ 315$ \\
& & \\
Ovarian Cancer & & \\
Intensive surveillance and prophylaxis & $\$ 7,981$ & $\$ 3,381$ \\
Population surveillance & $\mathrm{n} / \mathrm{a}$ & $\mathrm{n} / \mathrm{a}$ \\
\hline
\end{tabular}

Results of the cost-effectiveness analysis of genetic testing for familial breast cancer are shown in Figure 2. The intervention group was compared to control subjects for the period modelled (age 25-70). Costs incurred in the future have been discounted. 
Intervention effectiveness was measured by the number of years that the onset of cancer is delayed.

\section{Figure 2: Cost-effectiveness of genetic testing for familial breast cancer (BRCA1-BRCA2)}
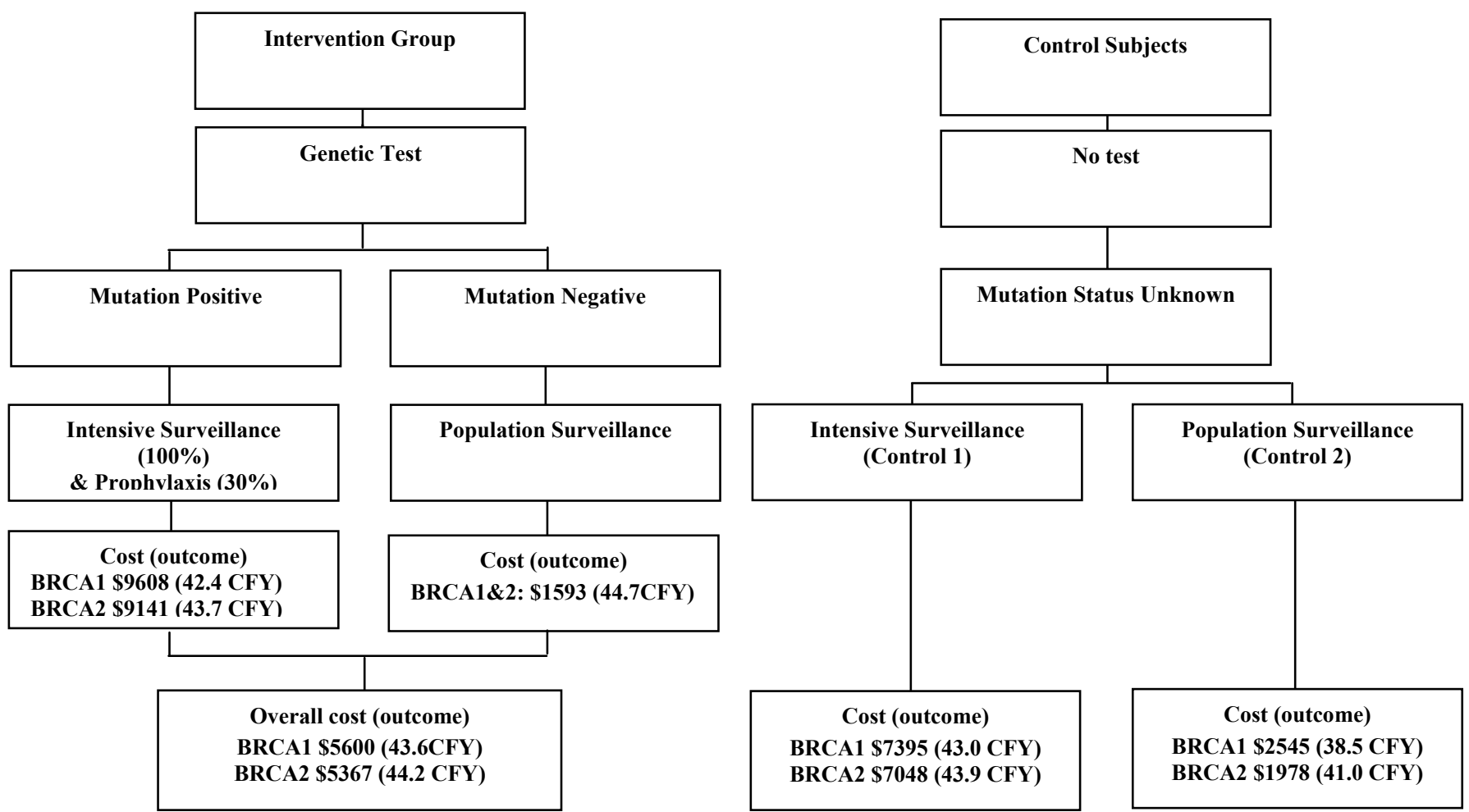

For each woman that undertook genetic testing for breast cancer:

- Compared to Control Group 1, BRCA1 intervention provided total net savings of \$1795 and delayed the onset of breast cancer by 6 months; BRCA2 intervention provided total net savings of \$1681 and the cancer onset was delayed by 3 months;

- Compared to Control Group 2, BRCA1 intervention incurred a \$3055 total net cost but delayed the onset of breast cancer by 5.1 years (\$601 per cancer-free year gained); BRCA2 intervention incurred $\$ 3389$ total net cost for a gain of 3.2 cancer-free years, (\$1070 per cancer-free year gained).

Compared to control subjects undergoing high-level surveillance since their mutation status is unknown, targeted BRCA1 intervention provided total net savings of $\$ 1795$ per woman and delayed the onset of breast cancer by 6 months. Likewise, BRCA2 intervention provided total net savings of $\$ 1681$ and delayed cancer by 3 months.

Compared to control subjects having population surveillance, BRCA1 intervention incurred a total net cost of $\$ 3055$ per woman but improved her outcome by 5.1 cancer-free years which is $\$ 601$ per cancer-free year gained. Similarly, BRCA2 intervention delayed the onset of breast cancer by 3.2 years, at a total net cost of \$3389 or, \$1070 for each cancer-free year gained.

The cost-effectiveness analysis of genetic testing for familial ovarian cancer is shown in Figure 3. 
Figure 3. Cost-effectiveness of genetic testing for familial ovarian cancer

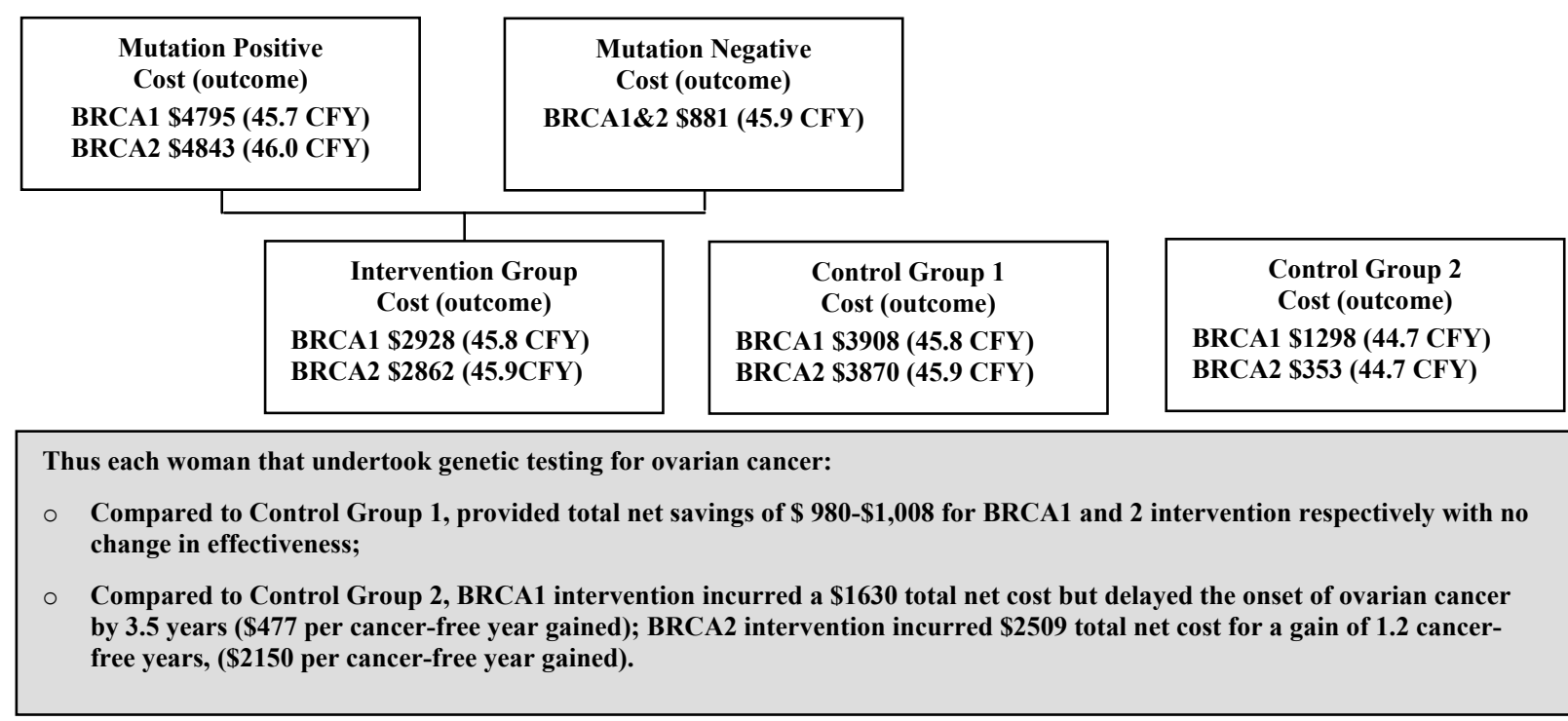

Compared to control subjects undergoing no surveillance, BRCA1 intervention incurred a $\$ 1630$ total net cost but delayed the onset of ovarian cancer by 3.5 years (\$477 per cancer-free year gained); BRCA2 intervention incurred \$2509 total net cost for a gain of 1.2 cancer-free years, ( $\$ 2150$ per cancer-free year gained).

\section{Sensitivity Analysis}

One-way sensitivity analysis was utilised to check the stability of the outcome given uncertainty in some variables, specifically the penetrance of BRCA mutations. Since this varied widely in the published literature ${ }^{36}$ cumulative cancer incidence by age 70 was explored using reported confidence intervals ${ }^{37}$. The results shown in Table 3 indicated that a higher level of penetrance would provide the intervention group with increased effectiveness (cancer-free years gained) for a lower net cost or greater net savings compared to no genetic testing.

Table 3: Impact of adjusted mutation penetrance on cost-effectiveness

\begin{tabular}{|lccccc|}
\hline \multicolumn{2}{|l}{ Cancer type } & Penetrance & $\begin{array}{c}\text { Cumulative } \\
\text { incidence }\end{array}$ & Net Saving & ${\text { Net } \text { Cost }^{2}}^{1}$ \\
\hline Breast: & BRCA1 & Low & $44 \%$ & $\$ 1687(3 \mathrm{mths})$ & $\$ 3581(3.2 \mathrm{yrs})$ \\
& & High & $78 \%$ & $\$ 1900(7 \mathrm{mths})$ & $\$ 2656(6.6 \mathrm{yrs})$ \\
& BRCA2 & Low & $31 \%$ & $\$ 1642(2 \mathrm{mths})$ & $\$ 3865(2.3 \mathrm{yrs})$ \\
& & High & $56 \%$ & $\$ 1719(4 \mathrm{mths})$ & $\$ 3403(3.9 \mathrm{yrs})$ \\
Ovarian: & BRCA1 & Low & $18 \%$ & $\$ 1011(0 \mathrm{mths})$ & $\$ 2289(1.8 \mathrm{yrs})$ \\
& & High & $54 \%$ & $\$ 1002(1 \mathrm{mth})$ & $\$ 1056(5.0 \mathrm{yrs})$ \\
& BRCA2 & Low & $2.4 \%$ & $\$ 993(1 \mathrm{mth})$ & $\$ 2765(6 \mathrm{mths})$ \\
& High & $19 \%$ & $\$ 1011(0 \mathrm{mths})$ & $\$ 2265(1.8 \mathrm{yrs})$ \\
\hline
\end{tabular}

\footnotetext{
${ }^{1}$ Compared to control subjects having intensive surveillance

${ }^{2}$ Compared to control subjects having population or no surveillance
} 
The estimated number of women that elect prophylactic bilateral mastectomy to prevent breast cancer in Western Australia (30\%) is low compared to the uptake in The Netherlands $(51 \%)^{38}$. The impact on cost-effectiveness of higher uptake was examined in the breast models (see Table 4). A greater uptake of prophylactic mastectomy in high-risk women compared to surveillance alone was found to increase the cost-effectiveness of genetic testing.

Table 4: Impact of prophylactic bilateral mastectomy uptake on costeffectiveness

\begin{tabular}{|lccl|}
\hline & Prophylactic uptake & Net Saving $^{1}$ & ${\text { Net } \text { Cost }^{2}}^{2}$ \\
\hline BRCA1 & $0 \%$ & $\$ 2125(1 \mathrm{mth})$ & $\$ 2725(4.6 \mathrm{yrs})$ \\
& $30 \%$ & $\$ 1795(6 \mathrm{mths})$ & $\$ 3055(5.1 \mathrm{yrs})$ \\
& $50 \%$ & $\$ 1574(9 \mathrm{mths})$ & $\$ 3276(5.4 \mathrm{yrs})$ \\
BRCA2 & & & \\
& $0 \%$ & $\$ 2081(0 \mathrm{mths})$ & $\$ 3179(2.9 \mathrm{yrs})$ \\
& $30 \%$ & $\$ 1691(3 \mathrm{mths})$ & $\$ 3569(3.2 \mathrm{yrs})$ \\
& $50 \%$ & $\$ 1432(5 \mathrm{mths})$ & $\$ 3828(3.4 \mathrm{yrs})$ \\
\hline
\end{tabular}

The impact of various discount rates $(0,3 \%, 5 \%$ and $7 \%)$ on the net cost or saving in the breast (BRCA1) intervention group was examined in Table 5.

Table 5: Discount rate effect on net cost or savings in the breast-BRCA1 intervention group

\begin{tabular}{|lcc|}
\hline Discount rate & Net Saving & Net Cost $^{2}$ \\
\hline No discount & $\$ 5028$ & $\$ 5012$ \\
Discount 3\% & $\$ 2687$ & $\$ 3550$ \\
Discount 5\% & $\$ 1795$ & $\$ 3055$ \\
Discount 7\% & $\$ 1232$ & $\$ 2724$ \\
\hline
\end{tabular}

The application of a 3\% discount rate instead of the 5\% rate utilised would increase per person net savings by $\$ 892$ or, increase the per person net cost by $\$ 495$ for the period observed, depending on the surveillance undertaken by control subjects. Findings were similar for the ovarian intervention group when the discount rate was adjusted (not shown).

\section{Discussion}

Early detection and intervention strategies resulting from predictive genetic testing for BRCA1/2 mutations in Western Australia has been demonstrated to be a costeffective use of resources under a range of scenarios.

Genetic testing enables the restriction of intensive surveillance to individuals with an identified BRCA1/2 gene mutation, leading to large net savings for the period observed (age 25-70). Compared with control subjects undergoing intensive surveillance and prophylaxis, the ovarian intervention group provided total net savings of $\$ 980-\$ 1008$ per woman. The breast intervention group provided total net 
savings of $\$ 1681-\$ 1795$ per woman and delayed the onset of breast cancer (6mths BRCA1, 3mths BRCA2).

Compared to control subjects undergoing only population surveillance, it was predicted the onset of cancer could be delayed in the genetic testing intervention group. For example, breast cancer could be delayed at a total net cost of $\$ 3055$ (5.1yrs) to $\$ 3389$ (3.2yrs) for women with identified BRCA1/2 mutations. This is a cost of $\$ 601$ or $\$ 1070$ per cancer-free year gained. Since population surveillance is not currently recommended for ovarian cancer, control subjects undergoing no surveillance were compared with women in the intervention group who were expected to delay the onset of ovarian cancer at a net cost of $\$ 1630$ (3.5yrs) to $\$ 2509$ (1.2years) for women with BRCA1/2 mutations. This is a cost of $\$ 477$ or $\$ 2150$ per cancer-free year gained.

These findings are consistent with a study by Tengs and Berry ${ }^{39}$ which found BRCA testing of high-risk women to be cost-effective, with estimated savings of $\$ 3400$ $\$ 4700$ per quality-adjusted life-year gained in a 30 -year-old woman. Grann et al ${ }^{40}$ also found genetic screening women to be cost-effective in Ashkenazi Jewish, but only if known mutation-carriers underwent the recommended prophylaxis. In the current study, genetic testing for BRCA mutations was found to be cost-effective even if women elected increased surveillance but declined surgery.

In addition to the reduction of cancer morbidity, benefits of predictive genetic testing include reduced anxiety from an unknown genetic background and the ability to make proactive decisions regarding medical and lifestyle options to prevent or minimise the risk of breast or ovarian cancer. The confirmation of risk status may also have important implications for family planning.

Although intangible costs and benefits were not explored in this study, aspects such as the psychological impact of extreme surgery, particularly bilateral mastectomy, on body image and sexuality warrants concern. However, a study by Hatcher et al found bilateral prophylactic mastectomy in women with high familial risk reduced psychological morbidity and anxiety and did not have a detrimental impact on women's body image or sexual functioning. They noted that women who chose such surgery had undergone more investigative tests than women who declined and had a higher, often inaccurate, perception of their risk of developing breast cancer ${ }^{41}$. Given the complexity of genetic risk communication this finding was not unexpected since confirmation as a BRCA mutation-carrier provides no certainty when, or if, cancer will occur in an individual, nor can a negative BRCA mutation result guarantee an individual will not develop cancer.

For clarity and comparison purposes the models were cancer and mutation specific. This represented a limitation of the study since some women may develop both breast and ovarian cancer and rarely carry both BRCA1 and BRCA2 mutations. We also assumed high-level patient compliance with clinical recommendations for intervention since the cost-effectiveness of genetic testing depends on compliance. 
The value of regular surveillance in women with a genetic or familial predisposition to breast cancer is uncertain ${ }^{42}$ although many studies have indicated a potential benefit for young women with a family history of cancer ${ }^{43,44,45,46}$. Other research suggested screening may be less effective since women with these mutations were more likely to develop cancer before menopause when breast tissue was dense, making it difficult to detect tumours on a mammogram ${ }^{47}$. Additionally the rate of growth of breast cancer is often faster in younger women, which can also decrease the effectiveness of screening at regular intervals ${ }^{48}$.

Findings by Kreige et $\mathrm{al}^{49}$ indicated magnetic resonance imaging was more sensitive than mammography for detecting beast cancers in women at increased risk because of inherited susceptibility. It is hoped that future research will provide clarity on the efficacy of intensive breast or ovarian cancer surveillance in known mutation-carriers.

The success of a genetic screening program however is dependent largely upon the compliance of clients with clinical recommendations for surveillance and the disclosure of mutation status by the proband to genetic relatives. In addition, the uptake of prophylactic surgery varies greatly between nations. In the Netherlands $51 \%$ of asymptomatic mutation carriers opted for bilateral mastectomy and 64\% for oophorectomy ${ }^{50}$ while the proportion of mutation-carriers that elect prophylaxis in Western Australia is around $30 \%{ }^{51}$.

According to Grann et $\mathrm{al}^{52}$ screening for BRCA mutations in the Ashkenazi Jewish population is only cost-effective if all women who tested positive underwent prophylactic surgery. However, this approach is complicated by prophylactic mastectomy not being totally protective, since breast cancer has been documented in women following prophylactic surgery ${ }^{53,54}$ and evidence that genetic information may even reduce motivation to change health behaviour ${ }^{55}$. Scheuer ${ }^{56}$ found that genetic counselling and testing increased compliance with surveillance and led to riskreducing operations and diagnosis of early-stage tumours in patients with BRCA1/2 mutations.

Investigation into the uptake of prophylactic surgery in Australia, the impact of cultural differences and support in the medical sector for such interventions may provide insight into levels of patient compliance with clinical recommendations and help shape future intervention protocols.

Early detection and intervention through predictive genetic testing for BRCA mutations in Western Australia has been demonstrated to be a relatively cost-effective use of resources under a range of scenarios, though further studies are needed to verify the results of long-term gains and, or costs from genetic testing. Additional research into compliance with clinical recommendations for surveillance, the disclosure of risk information to relatives and the degree of community support for such programs is required, since the cost-effectiveness of genetic testing will depend on the value of this information to patients and society. 


\section{Acknowledgements}

The authors wish to acknowledge the Familial Cancer Committee and Breast-Ovarian Cancer sub-Committee of the Department of Health Western Australia for their ongoing input and support and the below organisations for their contribution of data:

- Genetic Services and the Business Unit at the Women's and Children's Health Service, WA

- Gastro-Renal Business Unit, Sir Charles Gairdner Hospital, WA

- Division of Cancer \& Neurosciences, Royal Perth Hospital, WA

- Summary Measures Unit, Resources Division, Australian Institute of Health and Welfare

\footnotetext{
${ }^{1}$ National Health and Medical Research Council (NHMRC). 1999. Clinical Practice Guidelines Familial Aspects of Cancer: A Guide to Clinical Practice. Canberra, Australia.

${ }^{2}$ S. Goldie, S. and A. Levy. Genomics in medicine and public health: role of cost-effectiveness analysis. MSJAMA 2001; 286: 1637-1638.

${ }^{3}$ L. Scheuer et al. Outcome of preventive surgery and screening for breast and ovarian cancer in BRCA mutation carriers. J. Clin. Oncol. 2002; 20(5): 1260-1268.

${ }^{4} \mathrm{M}$. Tilanus-Linthorst et al. Earlier detection of breast cancer by surveillance of women at familial risk. Eur J Cancer 2000; 36(4): 514-519.

${ }^{5}$ L.C. Hartmann et al. Efficacy of bilateral prophylactic mastectomy in women with a family history of breast cancer. NEJM 1999; 340(2): 77-84.

${ }^{6}$ D. Haber. Prophylactic oophorectomy to reduce the risk of ovarian and breast cancer in carriers of BRCA mutations. NEJM 2002; 346(21): 1660-1662.

${ }^{7}$ R. Hogg and M. Frielander. Biology of epithelial ovarian cancer: implications for screening women at
} high genetic risk. J. Clin. Oncol. 2004; 22(7): 1315-1327.

8 N.D. Kauff et al. Risk-reducing salpingo-oophorectomy in women with a BRCA1 or BRCA2 mutation. NEJM 2002; 346(21): 1609-15.

${ }^{9}$ T.R. Rebbeck et al. Prophylactic oophorectomy in carriers of BRCA1 or BRCA2 mutations. NEJM 2002; 346(21): 1616-22.

${ }^{10}$ NHMRC op. cit. 1 .

${ }^{11}$ National Health and Medical Research Council (NHMRC). 2001. Clinical Practice Guidelines for the management of early breast cancer - 2nd Edition. Canberra, Australia.

${ }^{12}$ Australian Institute of Health and Welfare (AIHW). 2003. Health expenditure in Australia 2001-02. Number 17. Health and Welfare Expenditure Series No 4, Cat No HWE 8. Canberra, Australia.

${ }^{13}$ NHMRC op. cit. 10,11

14 Australian Institute of Health and Welfare (AIHW). 2004. Disease expenditure database unpublished. Canberra, Australia.

${ }^{15} \mathrm{~B}$. Meiser et al. Breast cancer screening uptake in women at increased risk of developing hereditary breast cancer. Breast Cancer Res Treat. 2000; 59: 101-111.

${ }^{16}$ Haber op. cit.6. pp.1660-1662.

${ }^{17}$ Rebbeck op. cit.9 pp.1616-22.

${ }^{18}$ Genetic Service of Western Australia (GSWA). 2003. Familial Cancer Program Service Audit. Perth, Department of Health Western Australia.

${ }^{19}$ AIHW op. cit.12.

${ }^{20}$ Australian Institute of Health and Welfare (AIHW). 2003. Cancer in Australia 2000. Cancer Series 23. AIHW \& Australasian Association of Cancer Registries, Canberra, Australia.

${ }^{21}$ A. Antoniou et al. Average risks of breast and ovarian cancer associated with BRCA1 or BRCA2 mutations detected in case series unselected for family history: a combined analysis of 22 studies. Am. J. Hum. Genet. 2003; 72: 1117-1130.

22 ibid

${ }^{23}$ National Health and Medical Research Council, National Breast Cancer Centre: Advice about familial aspects of breast cancer and ovarian cancer, a guide for health professionals, see: 
http://www.nbcc.org.au/bestpractice/resources/BOG_BreastOvarianGuideSimpl.pdf. Accessed 3 Aug 2005.

${ }^{24}$ GSWA op. cit 18 .

${ }^{25}$ Hartmann op. cit.5 pp.77-84.

${ }^{26}$ T. Rebbeck et al. Bilateral Prophylactic Mastectomy Reduces Breast Cancer Risk in BRCA1 and BRCA2 mutation Carriers: The PROSE Study Group. J. Clin. Oncol. 2004; 22(6): 1055-1062.

${ }^{27}$ International Agency for Research on Cancer of World Health Organisation (IARC). 2002. Press Release No139: Mammography screening can reduce deaths from breast cancer. Lyon, France.

${ }^{28}$ G.P. Gui et al. The incidence of breast cancer from screening women according to predicted family history risk: Does annual clinical examination add to mammography? Eur J Cancer 2002; 37(13): 1668-1673. Lyon, France.

${ }^{29} \mathrm{~J}$. Kollias et al. Screening women aged less than 50 years with a family history for breast cancer. Eur J Cancer 1998; 34(6): 878-883.

${ }^{30}$ R.D. Macmillan. Screening women with a family history of breast cancer - results from the British Familial Breast Cancer Group. Eur J Surg Oncol 2000; 26(2): 149-52.

${ }^{31}$ Tilanus-Linthorst op. cit.4 pp.514-519.

${ }^{32}$ I. Komenaka et al. The development of interval breast malignancies in patients with BRCA mutations. Cancer 2004; 100: 2079-2083.

${ }^{33}$ Hogg op. cit. 7 pp.1315-1327.

${ }^{34}$ Haber op. cit.6 pp.1660-1662.

${ }^{35}$ Rebbeck op. cit.9 pp.1616-22.

${ }^{36}$ C.B. Begg. On the use of familial aggregation in population-based case probands for calculating penetrance. J Natl Cancer Inst. 2002; 94(16): 1221-1226.

${ }^{37}$ Antoniou op. cit.21 pp.1117-1130.

${ }^{38}$ E.J. Meijers-Heijboer et al. Presymptomatic DNA testing and prophylactic surgery in families with a BRCA1 or BRCA2 mutation. Lancet 2000; 355: 2015-2020.

$39 \mathrm{~T}$. Tengs and D. Berry. The cost-effectiveness of testing for the BRCA1 and BRCA2 breast-ovarian cancer susceptibility genes. Disease Management and Clinical Outcomes 2000;1: 15-24.

40 V.R. Grann et al. Benefits and costs of screening Ashkenazi Jewish women for BRCA1 and BRCA2. J Clin Oncol 1999;17(2): 494-500.

41 M.B. Hatcher et al. The psychosocial impact of bilateral prophylactic mastectomy: prospective study using questionnaires and semi-structured. BMJ 2001; 322(7278): 76.

42 R. Calderon-Margalit and O. Paltiel. Prevention of breast cancer in women who carry BRCA1 or BRCA2 mutations: a critical review of the literature. Int J Cancer 2004; 112: 357-364.

43 Kollias op. cit.28 pp.878-883.

44 Tilanus-Linthorst op. cit.4 pp.514-519.

45 O. Meittenen et al. Mammographic screening: no reliable supporting evidence? The Lancet 2002; 359: 404-406.

$46 \mathrm{M}$. Kriege et al. Efficacy of MRI and mammography for breast-cancer screening in women with a familial or genetic predisposition. NEJM 2004; 351(5): 427-437.

47 Tilanus-Linthorst op. cit.4 pp.514-519.

48 D.S. Buist et al. Factors contributing to mammography failure in women aged $40-49$ years. J Natl Cancer Inst 2004; 96(19): 1432-40.

49 Kriege op. cit.45 pp.107-109.

50 Meijers-Heijboer op. cit.37 pp.2015-2020.

51 GSWA op. cit 18.

52 Grann op. cit.39 pp.494-500.

53 Hartmann op. cit.5 pp.77-84.

54 Rebbeck op. cit.25 pp.1055-1062.

55 T. Marteau and C. Lerman. Genetic risk and behavioural change. BMJ 2001; 322: 1056-1059.

56 Scheuer op. cit.3 pp.1260-1268. 